\title{
Correction to: A biometric cryptosystem scheme based on random projection and neural network
}

\author{
Jialiang Peng ${ }^{1,2} \cdot$ Bian Yang $^{3} \cdot$ B. B. Gupta ${ }^{4,5} \cdot$ Ahmed A. Abd El-Latif $^{6}$
}

Published online: 19 April 2021

(C) Springer-Verlag GmbH Germany, part of Springer Nature 2021

\section{Correction to: Soft Computing \\ https://doi.org/10.1007/s00500-021-05732-2}

While typesetting the article the affiliation to the authors are incorrectly published. The correctly authors and affiliation has been copied below.

The original article has been corrected.

The original article can be found online at https:// doi.org/10.1007/s00500-021-05732-2.

Jialiang Peng

jialiangpeng@hlju.edu.cn

$\triangle$ B. B. Gupta

Gupta.brij@gmail.com

Bian Yang

bian.yang@ntnu.no

Ahmed A. Abd El-Latif

a.rahiem@gmail.com; alatif@science.menofia.edu.eg

1 School of Data Science and Technology, Heilongjiang University, Harbin, China

2 Guangxi Key Laboratory of Cryptography and Information Security, Guilin, China

3 Department of Information Security and Communication Technology, Norwegian University of Science and Technology, Gjøvik, Norway

4 Computer Engineering Department, NIT Kurukshetra, Kurukshetra, India

5 Department of Computer Science and Information Engineering, Asia University, Taichung, Taiwan

6 Department of Mathematics and Computer Science, Faculty of Science, Menoufia University, Shebin El-Koom, Egypt
Publisher's Note Springer Nature remains neutral with regard to jurisdictional claims in published maps and institutional affiliations. 